\title{
Imaging Atherosclerosis and Vulnerable Plaque
}

\author{
Mehran M. Sadeghi ${ }^{1}$, David K. Glover ${ }^{2}$, Gregory M. Lanza ${ }^{3}$, Zahi A. Fayad ${ }^{4}$, and Lynne L. Johnson ${ }^{5}$ \\ ${ }^{1}$ Yale University School of Medicine, New Haven, Connecticut, and Veterans Administration Connecticut Healthcare System, West \\ Haven, Connecticut; ${ }^{2}$ University of Virginia, Charlottesville, Virginia; ${ }^{3}$ Washington University in St. Louis, St. Louis, Missouri; \\ ${ }^{4}$ Mount Sinai School of Medicine, New York, New York; and ${ }^{5}$ Columbia University, New York, New York
}

Identifying patients at high risk for an acute cardiovascular event such as myocardial infarction or stroke and assessing the total atherosclerotic burden are clinically important. Currently available imaging modalities can delineate vascular wall anatomy and, with novel probes, target biologic processes important in plaque evolution and plaque stability. Expansion of the vessel wall involving remodeling of the extracellular matrix can be imaged, as can angiogenesis of the vasa vasorum, plaque inflammation, and fibrin deposits on early nonocclusive vascular thrombosis. Several imaging platforms are available for targeted vascular imaging to acquire information on both anatomy and pathobiology in the same imaging session using either hybrid technology (nuclear combined with CT) or MRI combined with novel probes targeting processes identified by molecular biology to be of importance. This article will discuss the current state of the art of these modalities and challenges to clinical translation.

Key Words: clinical cardiology; molecular imaging; vascular; atherosclerosis; plaque; vascular remodeling

J Nucl Med 2010; 51:51S-65S

DOI: 10.2967/jnumed.109.068163

$\mathbf{T}$ he anatomy of vascular disease has been appreciated for centuries. More recently, with the explosive growth of molecular biology, the mechanisms for the common vascular diseases, including atherosclerosis, transplant graft vasculopathy, in-stent restenosis, and aneurysm formation, have been elucidated. Several anatomic features are common to all vascular lesions. Important among these is expansive or restrictive vascular remodeling. For expansive remodeling to occur, the extracellular matrix remodels by enzymatic degradation and cell apoptosis. Restrictive vascular remodeling occurs by neointimal formation. In transplant vasculopathy and in-stent restenosis, this process occurs by smooth muscle cell proliferation. In atherosclerosis, smooth muscle cells migrate from the adventitia and transform into monocytes or macrophages. Monocytes are also recruited from the circulation and become engorged

Received Oct. 15, 2009; revision accepted Jan. 15, 2010

For correspondence or reprints contact: David K. Glover, University of

Virginia Health System, P.O. Box 800500, Charlottesville, VA 22908-0500.

E-mail: dglover@virginia.edu

COPYRIGHT () 2010 by the Society of Nuclear Medicine, Inc. with lipids, producing large lipid-filled cores. To accommodate plaque growth, the extracellular matrix remodels by enzymatic degradation and apoptosis. Inflammation is a prominent feature of this process.

All of the stages along the way to advanced vascular disease involve biologic processes that can be targeted for imaging. Several imaging platforms are available for targeted vascular imaging and include nuclear, CT, MRI, and optical. This section will discuss the first 3 of these platforms. The described imaging modalities acquire information on both anatomy and pathobiology at the same time. This is achieved either by injecting a radiolabeled probe targeting the biologic process of interest and performing hybrid imaging-either SPECT/CT or PET/ CT - or by injecting a nanoparticle targeting the biologic process and performing MRI.

\section{MOLECULAR IMAGING OF VASCULAR REMODELING}

Vascular remodeling, defined as persistent changes in the structure or composition of blood vessels, is a common feature of vascular pathologies. Modalities that show the anatomy of blood vessels provide useful information, for example, on aortic aneurysm, but additional information on the remodeling process can enhance our understanding of pathophysiology, guide the selection and assess the efficacy of therapeutic interventions, and provide relevant information on prognosis.

Components of vascular remodeling, including both geometric remodeling (expansive or restrictive) and changes in the vessel wall composition (hypertrophy or hypotrophy), play roles in various vascular pathologies. In early atherosclerosis, in conjunction with plaque development and intimal thickening, the total vessel area increases (expansive remodeling) to maintain lumen size and blood flow. Over time, this expansive remodeling becomes insufficient or is replaced with constrictive remodeling limiting blood flow and resulting in ischemia. In apparent contradiction to its protective role in preventing ischemia, expansive remodeling in atherosclerosis has been linked to plaque vulnerability and acute coronary syndromes (1).

In graft arteriosclerosis, diffuse neointimal hyperplasia of epicardial coronary arteries and their distal branches 
leads to ischemia and organ loss. Despite major advances in the treatment of acute rejection, graft arteriosclerosis remains the main cause of late organ failure after cardiac transplantation. Coronary angiography and myocardial perfusion imaging, performed repeatedly on transplant patients, can detect only the late stages of graft arteriosclerosis, when therapeutic interventions are less effective. Intravascular ultrasound is able to detect early intimal hyperplasia. However, this is an invasive procedure, limiting frequent sampling.

Expansive remodeling is the main pathologic feature of aneurysm. In aortic aneurysm, focal expansion of the aorta, in conjunction with medial hypotrophy and mechanical hemodynamic forces, leads to aortic rupture or dissection, complications that are associated with high morbidity and mortality. Although size is the best predictor of aneurysm rupture, a large number of complications occur in smaller aneurysms that do not meet the criteria for surgical or intravascular repair. Rapid expansion of small aneurysms is believed to increase their risk of rupture or dissection. However, with existing imaging modalities (CT, MRI, ultrasound), aneurysm expansion can be detected only retrospectively through serial anatomic imaging.

In postangioplasty in-stent restenosis, as well as in vasculopathies associated with diabetes, hypertension, and chronic renal impairment, neointimal hyperplasia appears as the prominent pathologic feature. Vascular smooth muscle cell (VSMC) proliferation and migration and matrix remodeling are key cellular and molecular events in neointimal hyperplasia and may be detected by molecular imaging. Resting VSMCs in the media are in the so-called contractile phenotype. In response to injury or after exposure to growth factors, medial VSMCs lose contractile proteins and convert to the so-called proliferative or synthetic phenotype. Synthetic VSMCs have increased proliferation and migration capability and eventually form most of the cells in the neointima. VSMC phenotypic switch is associated with changes in membrane proteins and other antigens that can serve as targets for molecular imaging. One such molecule present on proliferating VSMCs is the antigen for Z2D3, an antibody used in the first molecular imaging studies of vascular remodeling (2).

Integrins, a family of heterodimeric membrane proteins involved in cell-cell and cell-matrix interaction, play an important role in cell proliferation, migration, and survival. Integrin $\alpha_{v} \beta_{3}$ has been extensively used as a target for molecular imaging of angiogenesis and other processes associated with cell proliferation. In addition to the expression level, integrin function is dependent on the activation state that occurs through changes in integrin structure altering the affinity for ligands. Given the ubiquitous expression of integrins, tracers with specificity for their high affinity, active conformation provide additional specificity to integrin targeting for molecular imaging in vivo. In injury-induced vascular remodeling, whether mechanical or immune-induced, $\alpha_{\mathrm{v}} \beta_{3}$ integrin is upregulated and activated in the media and neointima of remodeling arteries with a temporal pattern paralleling that of cell proliferation. RP748, an ${ }^{111}$ In-labeled quinolone with high affinity and specificity for activated $\alpha_{v}$ integrins, can track cell proliferation in vascular remodeling, as demonstrated by autoradiographic studies in murine models of vascular remodeling $(3,4)$. It remains to be empirically determined whether the integrin signal is sufficient for in vivo imaging.

Matrix remodeling, through matrix protein synthesis, contraction, and proteolytic degradation, is an integral feature of vascular remodeling. Proteases, including matrix metalloproteinases (MMPs), a large family of calcium- and zinc-dependent proteases, play an important role in both components of vascular remodeling. VSMC migration in neointimal hyperplasia is dependent on changes in the matrix that facilitate cell anchoring and movement. In geometric remodeling, protease-mediated matrix turnover is required for changes in the vessel scaffold. MMPs and other proteases, such as cathepsins, are key players in matrix remodeling. MMP protease activity is regulated by the expression level, activation state, and presence of tissue inhibitors. MMP-2 and tissue inhibitors 1 and 2 of MMPs have low expression in normal arteries. In response to injury, various proteases, including members of the MMP family, are upregulated and activated in the vessel wall. Inflammatory cells are a major source of activated MMPs in vascular disease. The important role of MMP expression in tumor growth motivated the development of broad-based metalloproteinase inhibitors (MPIs) that can be radiolabeled and used to track vascular remodeling in vivo. One such compound, a radiolabeled broad-spectrum MMPinhibitor, ${ }^{123}$ I-CGS 27023A, was first used to detect MMP upregulation after carotid artery ligation in apolipoprotein $\mathrm{E}$ knockout (apoE $\mathrm{E}^{-1-}$ ) mice (5). In a subsequent study, RP782, an ${ }^{111}$ In-labeled tracer with specificity for activated MMPs, localized to remodeling carotid arteries of apoE ${ }^{-1-}$ mice after wire injury (Fig. 1) (6). In this model of vessel injury, in which neointimal hyperplasia is the predominant histologic feature, MMP activation was detected by small-animal SPECT/CT and paralleled changes in vessel wall thickness. MMPs play a key role in the pathogenesis of arterial aneurysm, and their overexpression has been linked to aneurysm rupture. Small-animal SPECT of MMP activation in carotid aneurysm has been reported (7), and it remains to be empirically determined whether imaging MMP activation in aneurysm can help predict aneurysm expansion. Activation of MMPs also plays a major role in vessel remodeling in atherosclerosis and will be further discussed in that context below.

\section{MOLECULAR IMAGING OF ATHEROSCLEROSIS}

\section{inflammation}

Cardiovascular disease is the major cause of mortality and morbidity in developed countries, and atherosclerosis is responsible for many of the severe manifestations, including 

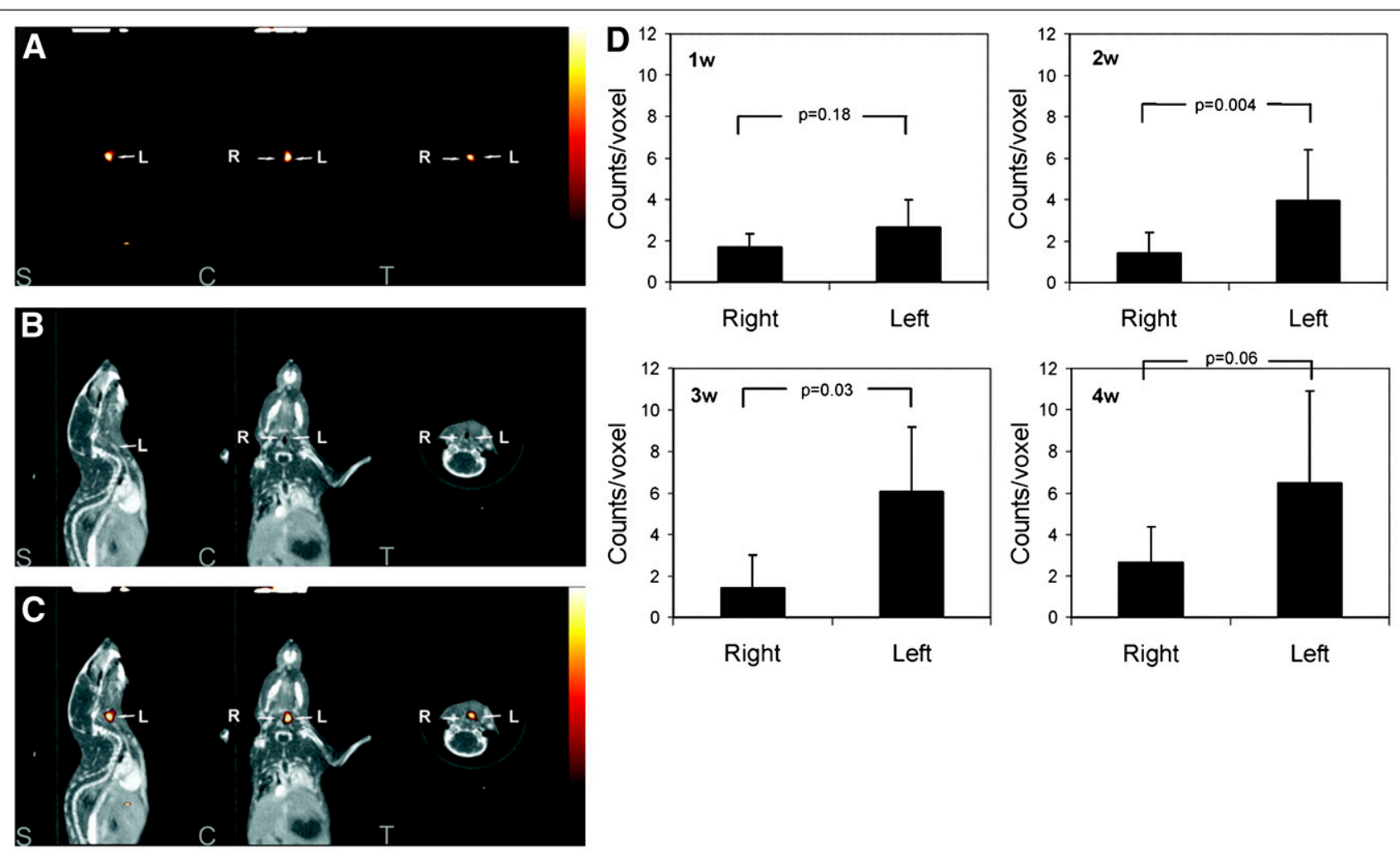

FIGURE 1. RP782 imaging of MMP activation in vascular remodeling. RP782 micro-SPECT (A), CTA (B), and fused microSPECT/CT (C) images at $3 \mathrm{wk}$ after left carotid injury in apoE ${ }^{-1-}$ mice demonstrate enhanced RP782 uptake in injured left as compared with control right carotid arteries. Quantification of carotid RP782 uptake at different time points after injury is shown in (D). S = sagittal slices; C = coronal slices; $\mathrm{T}=$ transverse slices; w = weeks. (Reprinted with permission of (6).)

myocardial ischemia, acute myocardial infarction, heart failure, and stroke. Detection of atherosclerosis with imaging has traditionally relied on the assessment of physical attributes of the vessel wall such as luminal narrowing that are present in late-stage lesions. However, the functional severity of lesions on angiography is not an accurate predictor of future cardiac events. It is now widely appreciated that atherosclerosis is a chronic and dynamic inflammatory disease. Inflammatory cells play a key role in all stages from initiation of plaque development to transition of a plaque from a stable to a rupture-prone state.

Several approaches have been evaluated for radioimaging the inflammatory process in atherosclerosis. Some of these approaches have included imaging the accumulation of radiolabeled low-density lipoprotein (LDL) in atheromatous lesions in animals $(8,9)$ and humans $(10)$, chemokine MCP-1 receptor expression (11), inflammatory cell trafficking with ${ }^{111}$ In-oxine labeled monocytes (12), macrophage density by phagocytosis of ${ }^{64} \mathrm{Cu}$-labeled nanoparticles (13), and uptake of ${ }^{18} \mathrm{~F}-\mathrm{FDG}$. The reason why ${ }^{18} \mathrm{~F}-\mathrm{FDG}$ can be used to image macrophages is that these cells have a high basal metabolic rate that is dependent on the transport of exogenous glucose as a substrate. When activated, the metabolic rate further increases, requiring additional uptake of glucose. Thus, the high glucose use by activated macrophages presents a target for the ${ }^{18} \mathrm{~F}$-labeled derivative of glucose. Several small clinical studies have demonstrated the feasibility of imaging inflamed atherosclerotic plaques using ${ }^{18} \mathrm{~F}-\mathrm{FDG}$ in humans. These studies will be discussed in detail in the clinical trials section of this article. In this section, the focus is on a new approach to imaging inflammation in atherosclerotic plaque that involves targeted molecular imaging of the lectinlike oxidized-1 (LOX-1) LDL receptor.

A high serum level of LDL cholesterol is a major risk factor for atherosclerosis. Oxidation of native LDL is an early process in atherogenesis $(14,15)$. Oxidized LDL causes endothelial dysfunction (16) and is taken up by scavenger receptors on macrophages, resulting in the formation of cholesterol-loaded foam cells (17). Oxidized LDL also facilitates thrombus formation by reducing fibrinolysis and by promoting procoagulant activity via induction of tissue factor expression (18), by reducing vasodilator species nitric oxide (19), and by altering anticoagulant tissue-plasminogen activator and its endogenous inhibitor, PAI-1.

Oxidized LDL exerts its effects by binding to scavenger receptors on macrophages and to the LOX-1 LDL receptor 
(20). LOX-1 is a membrane protein that belongs structurally to the C-type lectin family and is expressed in vascular endothelium and in vessel-rich organs. LOX-1 does not share homology with any of the known scavenger receptors for oxidized LDL found in macrophages. The expression of LOX- 1 is induced by tumor necrosis factor- $\alpha$ angiotensin II and shear stress in endothelial cells (21-23). LOX-1 is also expressed in macrophages and VSMCs (24).

Ishino et al. were the first to report successful in vivo imaging of LOX-1 using a ${ }^{99 m}$ Tc-labeled anti-LOX-1 antibody and planar imaging in Wattanabe hyperlipidemic rabbits (25). Atheromatous lesions were clearly visualized by planar imaging. Histologic analysis revealed the highest accumulation of the probe in grade IV atheroma, with less uptake of the probe observed in more stable lesions. Li et al. recently designed a multimodality imaging probe targeted to the LOX-1 receptor and validated and tested the feasibility of the imaging probe both in vitro and in vivo (26). The probe consists of a liposome shell decorated with either a nonspecific IgG (nIgG) antibody or a murine antiLOX-1 antibody, and various reporters, including gadolinium, ${ }^{111} \mathrm{In}$, and DiI fluorescence. They found that the LOX-1 probe bound specifically to atherosclerotic plaque in both apoE ${ }^{-1-}$ and LDL-receptor-deficient $\left(\mathrm{LDLR}^{-1-}\right.$ ) mice that had been fed a high-fat and -cholesterol diet for more than 16-20 wk. As can be seen in Figure 2, the probe was readily visible in the aortic arch on SPECT/CT images $24 \mathrm{~h}$ after injection. The in vivo results were confirmed by ex vivo phosphor plate and fluorescence imaging. They also demonstrated that the LOX-1 probe bound preferentially to the plaque shoulder region and was colocalized with known markers of plaque vulnerability including extensive LOX-1

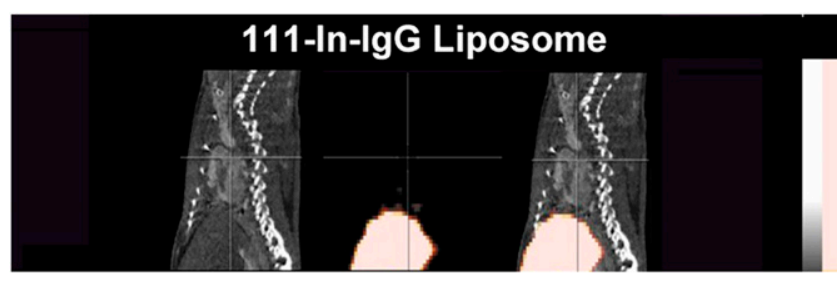

111-In-LOX-1 Ab Liposome

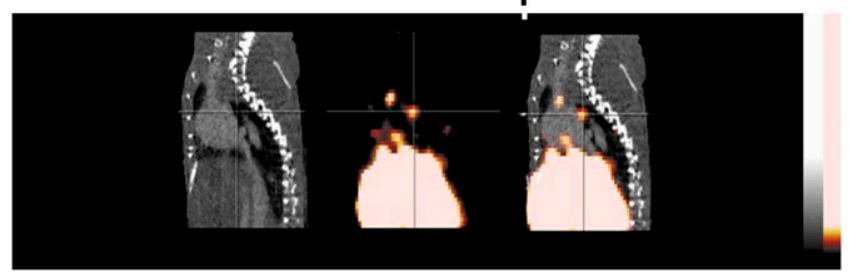

FIGURE 2. Contrast CT (left), micro-SPECT (center), and fused SPECT/CT (right) images of $\mathrm{apoE}^{-1-}$ mice fed Western diet for more than 16 wk. Imaging showed no focal aortic arch hot spots in mice injected with a nonspecific IgG antibody (nlgG) probe (top row), whereas all mice injected with targeted LOX-1 probe had hot spots in aortic arch (lower row). Results were confirmed by ex vivo phosphor imaging of excised aortas. Sudan IV staining demonstrated comparable plaques between the 2 groups. expression, macrophage accumulation, apoptosis, and MMP-9 expression.

\section{Apoptosis and the Metalloproteinases}

This section will discuss biologic targets, other than inflammation, that play a role in transformation of plaque stability: programmed cell death, enzymatic disruption of the extracellular matrix, and vessel remodeling. The anatomic features of acute plaque rupture are known from human autopsy studies on patients who died suddenly of acute coronary events (27-29). Immunohistochemical staining of sections taken through vulnerable plaques from patients, combined with experimental animal studies and advances in molecular biology, have identified apoptosis of macrophages infiltrated in the shoulder regions of thincapped fibroatheromas and increased expression of metalloproteinases (MMPs) as biologic markers of plaque vulnerability $(29,30)$. Segmental coronary artery dilation (positive remodeling) associated with large lipid-filled plaques is an anatomic feature associated with plaque vulnerability.

Imaging Apoptosis in Plaque. The biochemistry of apoptotic cell death involves activation of the caspase cascade (effector caspases 3 and 7) (30). Caspase 3 activation triggers both DNA fragmentation (identified by deoxyuride- $5^{\prime}$-triphosphate biotin nick end labeling [TUNEL] staining) and induces cell membrane alterations in cells undergoing apoptosis for phagocytic engulfment. This latter pathway has been used to target apoptosis of cancer cells using ${ }^{18} \mathrm{~F}$-labeled isatin sulfonamides (31). The caspase pathway triggers changes in cell membranes as a prelude to disruption and cell death. Phosphatidylserine is normally restricted to the inner layer of the phospholipid bilayer cell membrane. During apoptosis, phosphatidylserine is flipped to the outer bilayer. The naturally occurring protein annexin A5 avidly binds phosphatidylserine and was labeled first with a fluorescent probe and subsequently as a radiotracer for in vitro and in vivo imaging of apoptosis (32). The protein is linked to ${ }^{99 \mathrm{~m} T c}$ with bifunctional chelating agents such as hydrazinonicotinamide using an amide bond.

Programmed death can be triggered in any mammalian cell. Apoptosis plays a role in cardiomyocyte death during acute myocardial infarction, and annexin A5 has been used to image myocardial infarction (33). Annexin A5 has also been used to image atherosclerosis in several animal models, including high-fat-fed New Zealand White rabbits with aortic injury, apoE null and LDL-deficient mice, and domestic swine with coronary artery injury fed high-fat diets (34). In all these models, the target-to-background ratios were sufficient to permit in vivo visualization of the uptake of ${ }^{99 m} \mathrm{Tc}$ annexin $\mathrm{A} 5$ in areas of plaque identified at necropsy, and uptake correlated quantitatively with extent of apoptosis by caspase or TUNEL staining. In the mouse and rabbit studies, the atherosclerotic lesions were advanced, and staining for apoptosis colocalized to areas of 
macrophage infiltration (Fig. 3) (35). The signal from radiotracer uptake into atherosclerotic plaque can be used to monitor the effect of therapy to reduce apoptosis by administration of caspase inhibitors (36). However, in the porcine study only class II or III lesions were present and caspase-positive staining colocalized with smooth muscle cells. In earlier stages of plaque development, apoptosis of smooth muscle cells occurs as positive remodeling of the vessel occurs (37). This study in porcine coronary arteries showed that with a high ratio of target to background uptake, focal uptake of a radiotracer can be visualized on in vivo SPECT; the study also revealed the limited specificity of apoptosis imaging in identifying vulnerable plaque.

Imaging Metalloproteinase Expression in Plaque. The normal arterial wall media contains contractile VSMCs and a few resident macrophages. The extracellular matrix contains types I and III collagen; glycoproteins, including fibronectin, vitronectin, tenascin, and thrombospondin; and chondroitin/dermatan sulfate proteoglycans plus elastin $(30,38)$. During neointimal formation in early atherosclerosis, and as atherosclerotic lesions advance and the lipid core enlarges, the vessel wall positively remodels to accommodate neointima and preserve luminal area. As described previously, the remodeling process in the vessel wall occurs via breakdown of extracellular matrix through expression of the metalloproteinases (MMPs). With atherosclerosis, oxidized LDL increases MMP-1 and -3 expression (38). In addition to their catalytic effect, constitutive MMPs in VSMCs are induced by inflammatory cytokines, and their expression leads to migration and phenotypic modulation of macrophages (38).
In plaque monocyte-macrophages, MMP-9 is the most abundant gelatinase. Human tissue from aortic, carotid, and coronary arteries has correlated MMP expression with plaque vulnerability. Galis showed MMP-1, -3, and -9 in macrophages, VSMC, lymphocytes, and endothelial cells especially at the vulnerable shoulder region of plaques (39). A 2- to 4-fold increase in MMP-9 expression is found in human atherectomy tissue from patients with recent unstable versus stable coronary disease (40). Levels of MMP-1, MMP-3, MMP-8, and MMP-9 have been shown to be significantly greater in human atheromatous than in fibrous plaques (41).

As mentioned earlier, in vivo imaging of MMP expression can be achieved using radiolabeled broad-based MPIs (42-44). For example, a study in apoE null and LDLR null mice showed in vivo uptake of a ${ }^{99 \mathrm{~m}}$ Tc-labeled MPI RP805 in aortic atherosclerotic plaque (Fig. 4) (43). Uptake of radiotracer as percentage injected dose correlated with immunohistochemical staining for macrophages and with MMP-2 and MMP-9 (43). A change in the signal from the ${ }^{99 m}$ Tc-MPI in the plaque can be used to assess therapy to reduce MMP expression (44). In addition to inducing inflammatory cytokines, MMPs contribute to destabilizing plaque by segmental remodeling (4). Other platforms have been developed to image MMP expression, including optical imaging and MRI using an activatable near-infrared fluorescence probe and a gadolinium-coupled MPI $(45,46)$. In a direct comparison of the 2 radiotracers, imaging with ${ }^{99 m}$ Tc-labeled annexin A5 and ${ }^{99 m}$ Tc-MPI was performed on apoE null mice of different ages. Between 20 and $40 \mathrm{wk}$, as the aortic lesion area increased and the disease extended into the carotids, there were greater increases in percentage

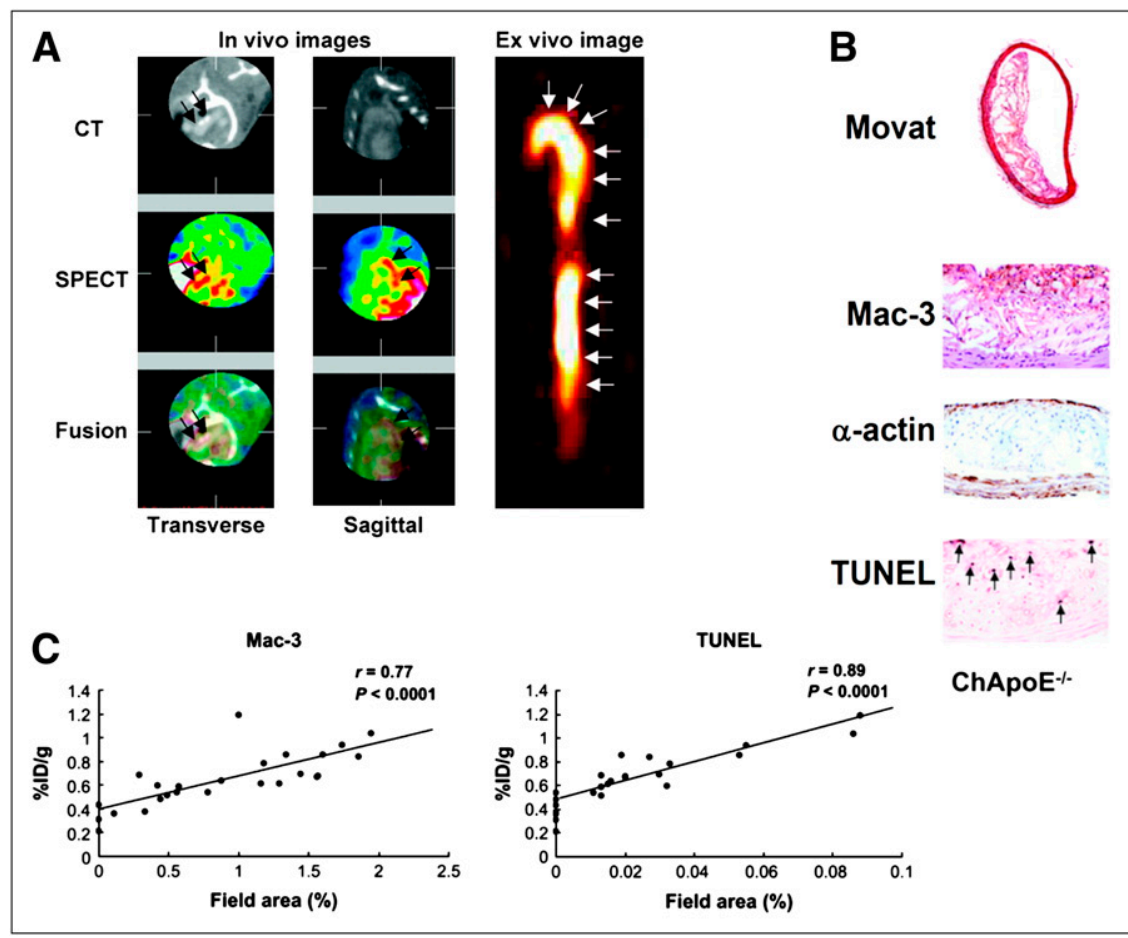

FIGURE 3. (A) Six reconstructed slices from in vivo hybrid small-animal SPECT/CT scan after injection of $99 \mathrm{mTC}$ annexin $\mathrm{AV}$ into $62-\mathrm{wk}$ apoE ${ }^{-/-}$mouse fed high-fat diet and showing uptake of tracer in aortic arch (red arrows). Image on right shows excised aorta imaged ex vivo. (B) Immunohistochemical stained sections through aorta shows American Heart Association class IV lesion with lipid core, prevalent macrophages, and TUNEL-positive nuclei. (C) Correlations between percentage injected dose (\%ID) and both macrophages and TUNEL-positive cells. (Reprinted from (35).) 


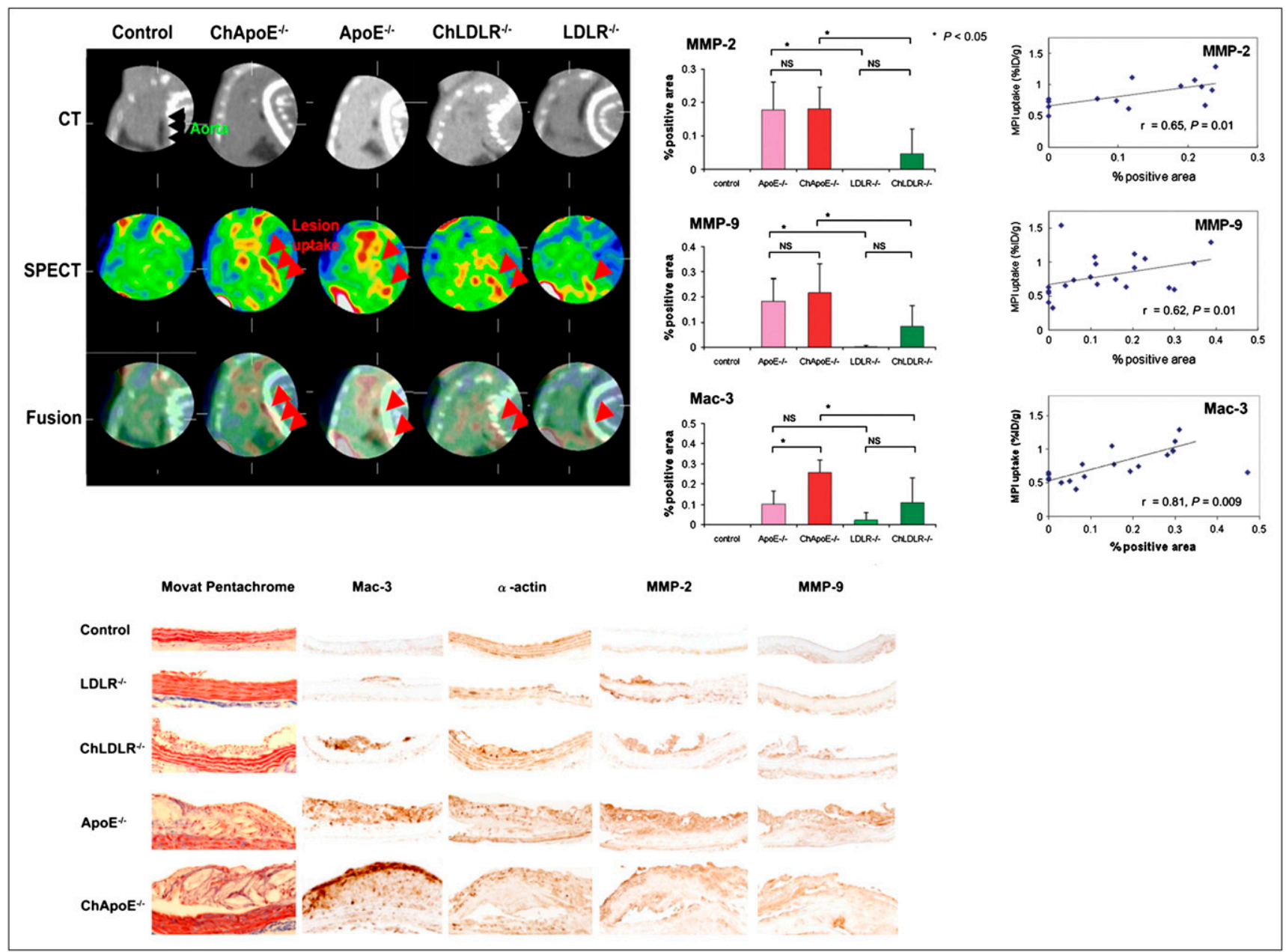

FIGURE 4. (Top left) Reconstructed slices from in vivo hybrid small-animal SPECT/CT scan after injection of $99 \mathrm{mT}$-labeled MPI into representative mice from 5 groups: control, apoE ${ }^{-/-}$fed high-fat diet $\left(C h A p o \epsilon^{-1-}\right)$, apoE ${ }^{-/-}$fed normal chow, LDLR ${ }^{-/-}$ fed high-fat diet (ChLDLR ${ }^{-1-}$ ), and LDLR $^{-1-}$ fed normal diet. Black arrows identify aorta, and red arrows identify tracer uptake in aortic arch (on SPECT and fused SPECT/CT), with greatest amount seen in apoE ${ }^{-1-}$ mouse fed high-fat diet. Scans of control mouse are negative. (Top right) Quantitative histologic analysis of MMP-2, MMP-9, and macrophages (Mac-3) for 4 groups of atherosclerotic mice, and control. (Bottom) Histopathologic and immunohistochemical staining of sections from aortae from 5 groups. (Reprinted from (43).)

MMP-2 and -9 than of percentage caspase-positive cells, indicating that MMP expression is greater than apoptosis as the disease progresses. These differences in histology correlated with differences in tracer uptake, and the results support the premise that radiolabeled MPI is a better imaging agent for more advanced disease than annexin A5 (47).

A radiotracer such as ${ }^{99 \mathrm{~m}} \mathrm{Tc}-\mathrm{MPI}$ that targets both macrophages and vessel remodeling has potential to noninvasively visualize vulnerable coronary lesions. Unlike ${ }^{18} \mathrm{~F}-\mathrm{FDG},{ }^{99 \mathrm{~m}} \mathrm{Tc}-\mathrm{MPI}$ shows little or no myocardial uptake in animal models of atherosclerosis and thus would allow a good target-to-background ratio if the signal from the lesions is robust enough to be seen on in vivo imaging. Because such an agent would have applications in both cancer and atherosclerosis, motivation by industry for drug development should be fairly high. A labeled compound that could be used in both PET and SPECT would have more widespread application.

\section{NANOPARTICLES WITH MRI AND SPECTRAL CT PLATFORMS TO IMAGE ATHEROSCLEROSIS}

The term molecular imaging has an expanded meaning to encompass biomedical diagnostics, noninvasive imaging, and targeted therapies related to pathologic molecular biosignatures. Over the last decade, research publication and patent activities involving nano-scaled technologies in the health sciences field have exponentially proliferated, reflecting the leadership role played by the National Institutes of Health through the National Cancer Institute's Unconventional Innovation Program, the Alliance for Nanotechnology in Cancer Program, and related nanoplatform partnerships in parallel with the National Heart, Lung, 
and Blood Institute Program for Excellence and related request-for-application funding initiatives.

\section{Superparamagnetic Nanoparticles}

One of the earliest applications of nanotechnology in MRI involved the use of paramagnetic iron oxide particles. Iron oxide crystals have long been used as superparamagnetic T2* contrast agents for MRI (48-51). Superparamagnetic iron oxide (particle diameter $>50 \mathrm{~nm}$ ) and ultrasuperparamagnetic iron oxide (USPIO, particle diameter $<50 \mathrm{~nm}$ ) particles have nonstoichiometric microcrystalline magnetite cores and are typically coated with dextran (e.g., ferumoxide) or siloxane (e.g., ferumoxsil) (52). Spontaneous phagocytic uptake of superparamagnetic iron oxide and USPIOs by macrophages in atherosclerotic plaque was recognized and demonstrated in 2000 and 2001 by Schmitz et al. and Ruehm et al. in hereditary or dietinduced hyperlipidemic rabbits (53-56). In 2003 this finding was extended to include human plaque (57). Systematic evaluation of USPIO-enhanced MRI contrast in carotid atheroma confirmed that the optimal signal intensity was achieved 24-36 h after administration. Subsequently, the USPIO compound ferumoxytol was compared with ferumoxtran-10 as a marker of macrophage activity in atherosclerotic plaques. Although both were reported to be effective, ferumoxytol had optimal luminal signal intensity $3 \mathrm{~d}$ after treatment, and ferumoxytol-treated rabbits had peak measurements $5 \mathrm{~d}$ after injection (58). Recently, new MRI pulse sequences and image postprocessing techniques have been developed to reverse the dark contrast appearance into a bright positive-contrast effect (59-66).

\section{Ligand-Directed Targeting of Iron Oxides}

The development of monocrystalline iron oxide nanoparticles helped to extend iron oxide MRI beyond the limitations of passive targeting through tissue accumulation and particle phagocytosis to ligand-directed or active targeting. Monocrystalline iron oxide nanoparticles have an average core diameter of $3 \mathrm{~nm}$ and can be directly coupled to homing ligands that specifically target epitopes in the tissue of interest (67). The targeting efficiency of iron oxide particles improved further with the development of dextran cross-linked iron oxide particles (68). Cross-linked iron oxide has been used with a variety of ligands, and although these particles may be demonstrated with histology to target tissue specifically soon after injection, detection on MRI remains delayed because of slow particle clearance into macrophages and nonspecific particle diffusion within tissue.

Recently, the colloidal iron oxide nanoparticle theranostic platform has been reported as a vascular constrained T1weighted molecular imaging agent that avoided typical magnetic bloom artifacts, permitted rapid in vivo molecular imaging without blood pool magnetization interference, and supported targeted drug delivery (69). Colloidal iron oxide nanoparticle offers rapid clearance $(<60 \mathrm{~min})$ of circulating interference on $\mathrm{T} 1$ contrast, whereas blood $\mathrm{T} 2$ shortening persists well over $2 \mathrm{~h}$, as expected for superparamagnetic agents. Moreover, colloidal iron oxide nanoparticle is designed for therapeutic drug delivery, for example, fumagillin, via a unique mechanism termed contact-facilitated drug delivery.

\section{Paramagnetic Nanoparticle Imaging}

In 1998, Sipkins demonstrated in vivo imaging of angiogenesis with paramagnetic polymerized liposomes in the VX2 tumor model (70) and Lanza et al. (71) demonstrated fibrin imaging with paramagnetic perfluorocarbon nanoparticles. Alternative nonparticulate approaches to molecular MRI were developed to target epitopes such as HER2/ neu receptors, using an avidin conjugated to gadoliniumdiethylenetriaminepentaacetic acid (12.5 gadolinium atoms per avidin), and fibrin in thrombus, targeted by a fibrinbinding peptide derivatized with 4 or 5 gadolinium atoms (72). Integrin-targeted liposome constructs were reported by Mulder et al. for angiogenesis-imaging rodent cancer models (73-75), and a paramagnetic lipoprotein analog was demonstrated for macrophage imaging in atherosclerotic plaque by Frias et al. and Lipinski et al. $(76,77)$.

Since 1998, the laboratory of Lanza et al. has extensively studied and refined ligand-targeted paramagnetic liquid perfluorocarbon nanoparticles for molecular imaging and targeted drug delivery in atherosclerosis $(71,78-83)$. Atherosclerotic plaque progresses from an early atheromatous lesion to a thin-capped vulnerable plaque through aggressive inflammatory and immune responses, comprising macrophage infiltration with necrotic core enlargement, neovascular expansion of the vasa vasorum, intraplaque hemorrhage $(84,85)$, and increased plaque angiogenesis.

Pathologic data from excised carotid arteries in patients treated for 3 mo with statins revealed a reduction in microvascular density, which was proposed as an explanation for the additional benefit of statins (86). Some have suggested that statins prune the plaque neovasculature, reducing intraplaque hemorrhage (a potential accelerator of atherosclerotic progression) and promoting plaque stabilization $(87,88)$. In a series of nanomedicine studies conducted on hyperlipidemic rabbits, $\alpha_{v} \beta_{3}$-targeted paramagnetic perfluorocarbon nanoparticles were shown to provide serial quantification of aortic angiogenesis (89), to deliver and monitor acute antiangiogenic therapy in early atherosclerosis (Fig. 5) (82), and to function synergistically with atorvastatin therapy to reduce plaque neovasculature and sustain the potentially stabilizing antiangiogenic benefit (83).

In the later stages of the atherosclerosis, perfluorocarbon nanoparticles can be used to detect and quantify the rich fibrin deposits of intravascular thrombus (78-80), the proximate cause of stroke and myocardial infarction. The high core density of fluorine, an excellent element for MR spectroscopy and MRI with no inherent background, supports MRI colocalization of the fluorine and proton 
FIGURE 5. (A) Time-of-flight MR angiogram $30 \mathrm{~min}$ after balloon stretch injury shows patent femoral arteries. Left artery was treated with $\alpha_{\mathrm{v}} \beta_{3}$-integrin-targeted paramagnetic nanoparticles with rapamycin, and saline was used for right artery. ( $B$ and $C$ ) MR angiograms 2 wk after injury and treatment, with arrows identifying regions of intraluminal plaque caused by balloon overstretch injury. In B, right artery, which has arterial plaque, was treated with $\alpha_{\mathrm{v}} \beta_{3}$-integrin-targeted nanoparticles without drug, and widely patent left artery was treated with $\alpha_{v} \beta_{3}$-integrin-targeted nanoparticles with rapamycin. In C, widely patent right artery was treated with $\alpha_{v} \beta_{3}$-integrin-targeted nanoparticles with rapamycin, and partially occluded left artery was treated with nontargeted nanoparticles with rapamycin. (D and E) Graphs of average (D) and maximum average (E) stenosis within injured and treated femoral arteries of New Zealand White rabbits 2 wk after balloon injury. Arterial segments were flash-frozen in optimal-cuttingtemperature compound, and alternate 7- $\mu \mathrm{m}$ sections were used for morphologic analysis (hematoxylin and eosin staining). (F) Area at risk of injured endothelium quantified on vascular en face preparations stained with Carstair stain. Normal, uninjured endothelium is yellow, and injured endothelium with fibrin deposition is red. (G) Quantitation of injured endothelium in area at risk $(100 \%=1-\mathrm{cm}$ excised vessel segment). Digitized images were analyzed on areas that had undergone balloon overstretch injury and were treated with $\alpha_{\mathrm{v}} \beta_{3}$-integrin-targeted nanoparticles with 0.4 mol\% rapamycin $(n=12)$ or saline control $(n=12)$. Vessels were excised on postinterventional days $1,7,14$, and 28 ( $n=3$ per group and time point). (Adapted with permission of (91).)
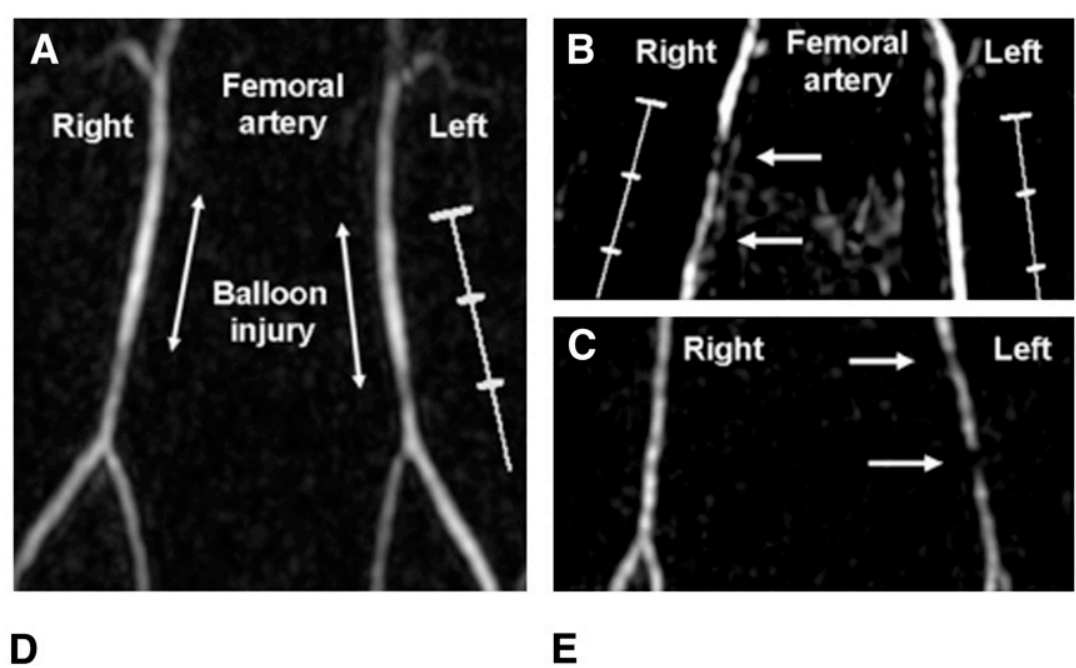

E
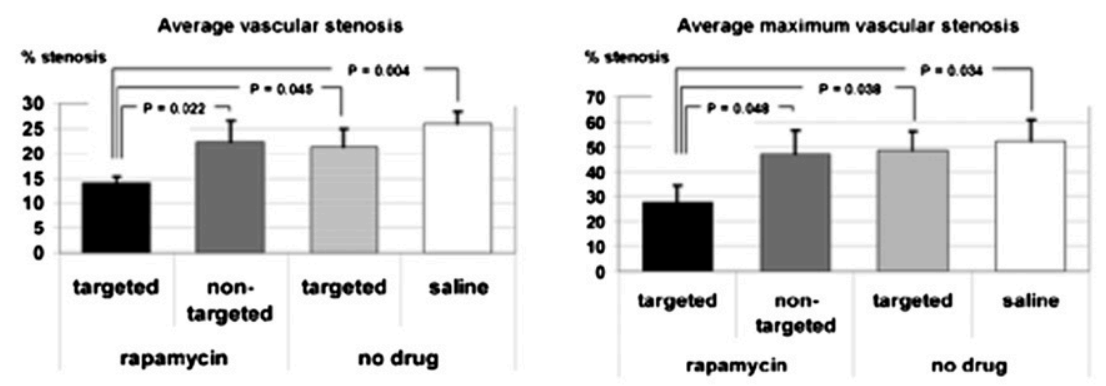

$\mathbf{F}$

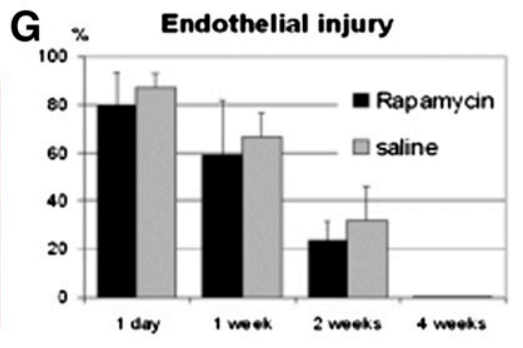

signal to confirm noninvasively the detection of perfluorocarbon nanoparticles and to quantify the number of bound nanoparticles, which indirectly assesses the extent of intraluminal clotting. Moreover, when angioplasty is required for revascularization, collagen III- and integrin-targeted paramagnetic nanoparticles have been used to detect and map intramural injury patterns in pigs (90), as well as to deliver rapamycin to inhibit restenosis in rabbits without impairing reendothelialization (Fig. 6) (91).

\section{Spectral CT Molecular Imaging}

A new field of CT molecular imaging is emerging with the development of novel nanotechnologies capable of delivering high metal payloads. CT continues to evolve from simple single-slice machines to multidetector arrays (e.g., 16, 64, or 256 slices) with concomitant improvement in CT tissue characterization through the development of dual-energy (i.e., 2-color) and now multicolored or spectral CT (92-95). Unlike the simple $\mathrm{x}$-ray attenuation of CT or the lowresolution differential absorption of dual-energy techniques, Spectral CT recognizes the k-edge of metals, which occurs when the attenuation of photons interacting with a $\mathrm{k}$-shell electron suddenly increases because of photoelectric absorption. Spectral CT scanners generate the traditional CT image and simultaneously acquire quantitative k-edge image data based on unique spectral footprints of specific elements, for example, gold, gadolinium, or bismuth. Iodine-based imaging agents will probably not be useful for clinical spectral CT because of a low k-edge energy, high internal scattering, and beam-hardening effects (i.e., depletion of $\mathrm{x}$-rays with higher attenuation coefficients from a polychromatic beam). Similarly, the use of metal crystals, which may have strong spectral CT contrast, will probably have inadequate bioelimination qualities. The 


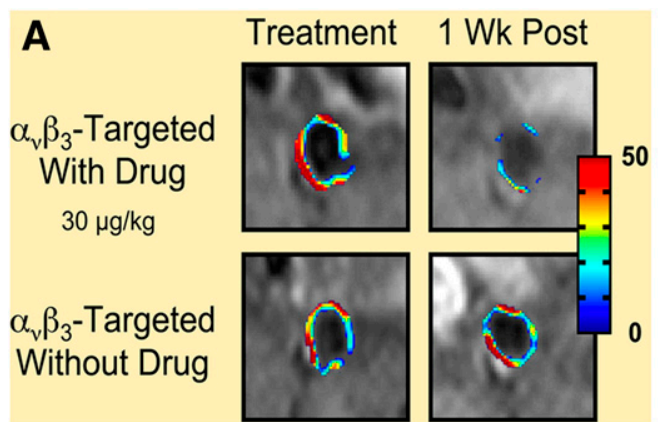

C

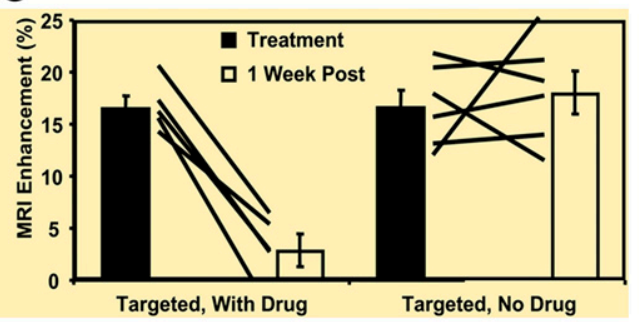

B

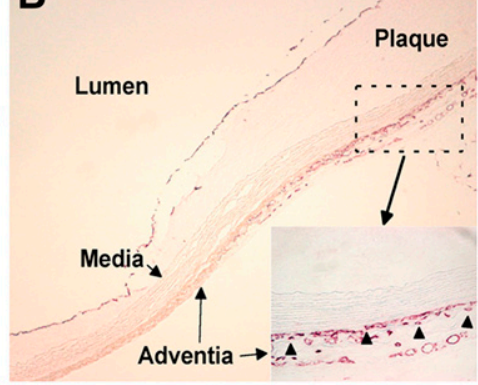

D

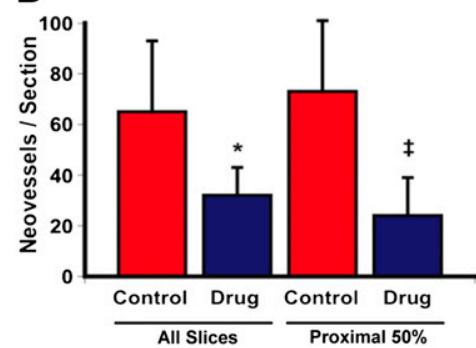

FIGURE 6. (A) MRI of abdominal aorta shows false-colored overlay of percentage signal enhancement at time of treatment (left) and $1 \mathrm{wk}$ after treatment (right). (B) Platelet endothelial cell adhesion molecule (PECAM)-stained section $(\times 4)$ of abdominal aorta from hyperlipidemic rabbit shows adventitia, media, and plaque. Higher-magnification inset $(\times 20)$ shows that microvessels were predominantly in adventitia associated with thickening neointima. Neovessels were generally not in regions where plaque progression was minimal or nonexistent in this cohort of rabbits. Arrowheads illustrate type of PECAM microvessels counted within each section to assess fumagillin antiangiogenic effects. Larger, mature vessels positively staining for PECAM were not included in these estimates. (C) Graph of aortic MRI signal enhancement averaged over all imaged slices at time of treatment (black bars) and 1 wk after treatment (white bars). Solid lines indicate individual animal's response to treatment over 7-d period. (D) Graph showing that number of neovascular vessels within adventitia was reduced $\left({ }^{\star} P<0.06\right.$; $\left.{ }^{\ddagger} P<0.05\right)$ in fumagillin-treated rabbits over proximal half of aorta (i.e., renal artery to diaphragm), which correlated with region of greatest MRI signal and fumagillin response in imaging studies. (Adapted with permission of (82).)
Lanza group has developed a family of "soft" metal nanocolloid k-edge agents that can be homed to fibrin fibrils within an intravascular thrombus or other suitable biomarker, to provide the location and concentration of targeted k-edge material. Fusion of these images with anatomic multislice-CT images permits localization of hot spots from intraluminal fibrin to the coronary bed.

\section{MOLECULAR IMAGING OF ATHEROSCLEROSIS IN CLINICAL TRIALS}

\section{Multimodality Imaging}

A clinically useful approach to atherosclerosis imaging involves the interrogation of several vascular beds in the same imaging session, such as aorta, carotid artery, and coronary arteries. To accomplish this, it is necessary to localize molecular probes to specific vascular sites. PET/ CT or SPECT/CT and MRI provide platforms to accomplish this task. Noninvasive quantification of inflammation can be performed with both of the nuclear imaging techniques-SPECT and PET. The radioactive tracer is administered intravenously and allowed to circulate within the body until it accumulates at the site of interest. On the basis of the rate of blood pool clearance, the time from injection to imaging is selected to allow blood levels to become sufficiently low to generate a favorable target-tobackground signal. Both SPECT and PET have sensitivities for the detection of molecular targets within the picomolar range, translating into the ability to use small doses of contrast agent, compared with MRI and CT. Nuclear imaging sensitivities compare favorably with both MRI and especially CT, which have sensitivities up to a trillion times lower (Fig. 7). The superior spatial resolution of PET (4-5 mm) makes it more attractive than SPECT (10-15 $\mathrm{mm}$ ). However, the spatial and temporal resolution of both methods is significantly less than that achieved by either MRI or CT. The high sensitivity of nuclear methods coupled with the favorable resolution of CT and MRI is the driver behind hybrid imaging systems such as PET/CT and PET/MRI that are now becoming available.

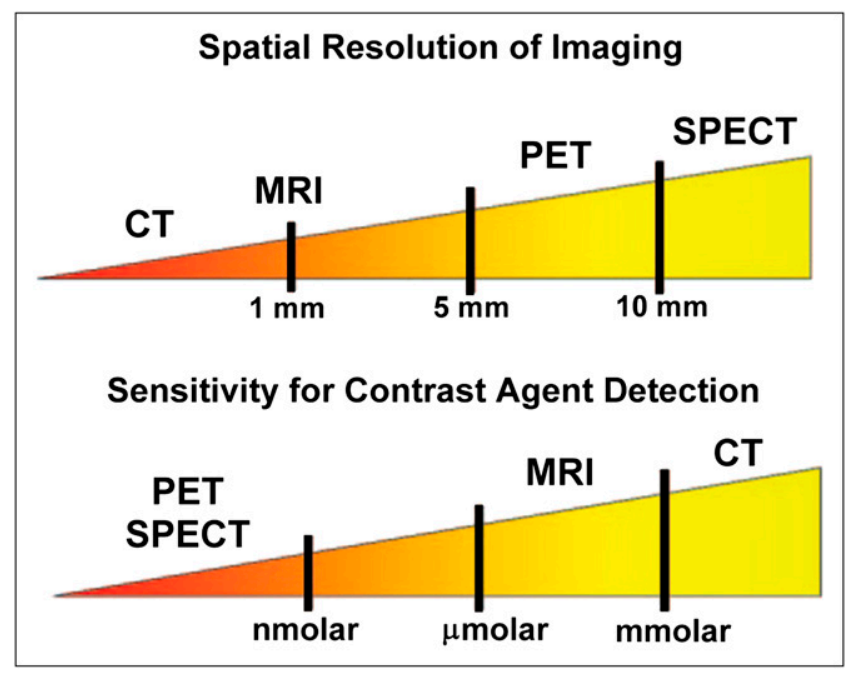

FIGURE 7. Illustration of relative spatial resolution of common imaging techniques, along with their sensitivities. 
TABLE 1. Comparison of Noninvasive Assessment of Atherosclerotic Plaques

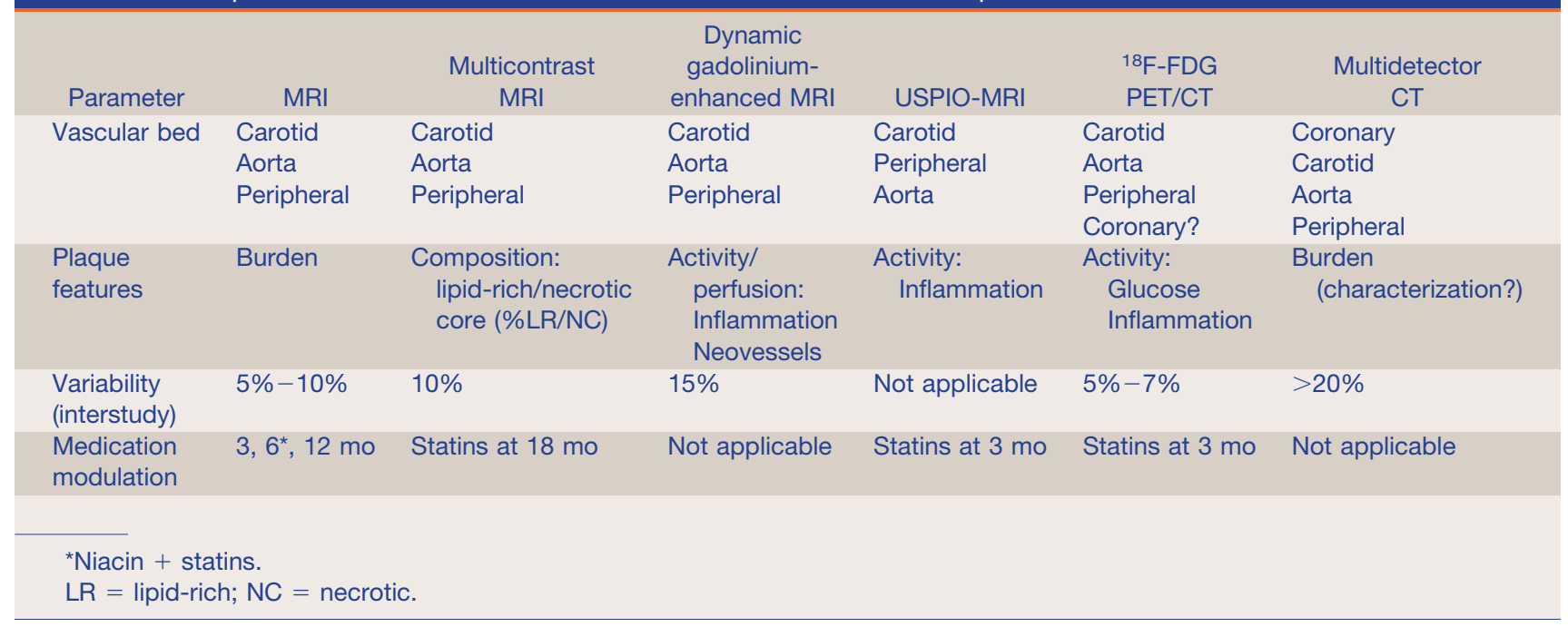

Some of the imaging techniques such as ${ }^{18}$ F-FDG PET, dynamic contrast-enhanced MRI $(96,97)$, and USPIO-enhanced MRI $(98,99)$ are close to the clinical arena (Table 1). Ongoing prospective trials will determine the place of imaging inflammation in predicting clinical events. Described below is a summary on the use of ${ }^{18} \mathrm{~F}$-FDG PET/CT and USPIO-MRI in atherosclerosis.

\section{Clinical Trials with ${ }^{18} \mathrm{~F}-\mathrm{FDG}$}

Identifying patients at high risk for an acute coronary event is clinically important. The Framingham Risk Score, which is used to project a 10-y risk from cardiovascular disease, is calculated on the basis of clinical and laboratory parameters: age, sex, total and HDL cholesterol, smoking history, and systolic blood pressure. The accuracy of the Framingham Risk Score in discriminating risk is approximately $75 \%$. However, some patients identified as being at low risk for mortality in the next $10 \mathrm{y}$ by the Framingham Risk Score actually have an increased lifetime risk of coronary artery disease events. Screening large numbers of patients with a costly and time-consuming imaging procedure to find the small number at highest risk is impractical and impossible in an era of cost containment. A biomarker measured from a simple blood sample would be the most cost-effective approach. C-reactive protein is a widely available biomarker to discriminate the degree of risk in this patient population. The recent JUPITER trial demonstrated that patients with normal cholesterol levels, but elevated plasma $\mathrm{C}$-reactive protein levels, had their risk of an event cut in half in just $2 \mathrm{y}$ by aggressive statin therapy (100). However, administering aggressive statin therapy to all patients who meet those criteria would be expensive, and the long-term safety of aggressive statin therapy is unknown. Another approach would be to identify a high-risk group based on risk factors and biomarkers and on this select group perform a more expensive imaging procedure that would identify either plaque morphologic features or biologic signals associated with plaque vulnerability.

Inflammation is important at many stages of atherosclerotic plaque development (101). As mentioned previously, ${ }^{18} \mathrm{~F}$-FDG PET is a molecular imaging technique that is highly sensitive to metabolically active processes that use glucose as a fuel, such as the macrophage foam cells within atherosclerosis. ${ }^{18} \mathrm{~F}-\mathrm{FDG}$ imaging is performed on a combined PET/CT system. The anatomic information from the CT scan is used to localize ${ }^{18} \mathrm{~F}-\mathrm{FDG}$ uptake to the vascular tree.

${ }^{18} \mathrm{~F}-\mathrm{FDG}$ uptake in arterial walls was first noted in the aorta of patients undergoing PET for cancer staging $(102,103)$. It was soon discovered that the extent of ${ }^{18} \mathrm{~F}-$ FDG uptake was greater in older patients (102-104) and those with cardiovascular risk factors (105-107). Since these early studies, it is now established that ${ }^{18} \mathrm{~F}-\mathrm{FDG}$ uptake is generally greater in symptomatic atheromatous plaques than in asymptomatic lesions (108). Additionally, the arterial ${ }^{18}$ F-FDG signal is linked to levels of inflammatory biomarkers (109) and to the number of components of the metabolic syndrome (110). More recently, it has been demonstrated that arterial ${ }^{18} \mathrm{~F}-\mathrm{FDG}$ signal can be reduced by either drug therapy (111) or dietary and lifestyle changes (112).

Arterial ${ }^{18} \mathrm{~F}-\mathrm{FDG}$ PET/CT is currently being applied in the assessment of novel antiatherosclerosis drugs, in which direct evidence of an antiinflammatory effect on the artery wall is useful (Clinical Trials.gov reveals 18 ongoing studies as of September 2009). Early evidence of inflammation reduction can potentially avoid the need for lengthy, costly outcome studies for drugs that are not sufficiently potent.

Imaging inflamed atheroma in the coronary vasculature with ${ }^{18} \mathrm{~F}-\mathrm{FDG}$ is considerably more challenging than in the 
carotid artery or aorta because of myocardial uptake of ${ }^{18} \mathrm{~F}$ FDG and the smaller size of the coronary arteries. A recent study demonstrated the feasibility of imaging inflamed lesions in the coronary vessels using PET/CT by first suppressing myocardial ${ }^{18} \mathrm{~F}-\mathrm{FDG}$ uptake by having the patient consume a high-fat, low-carbohydrate diet (113).

\section{Clinical Trials with USPIO-MRI}

USPIO-MRI has been shown to identify inflammatory changes by monitoring macrophage uptake, a major component of high-risk (vulnerable) plaques. To date, no published study has shown correlations between the dose of prescribed statin and in vivo changes in macrophage distribution. "Atorvastatin Therapy: Effects on Reduction of Macrophage Activity (ATHEROMA)" is the first prospective molecular MRI study to correlate the in vivo effects of statin therapy on carotid plaque inflammation as observed by MRI (114). The results of the study found a significant reduction from baseline in USPIO-enhanced MRI-defined plaque inflammation in the high-dose atorvastatin group at both 6 and 12 wk after treatment. Such changes were not observed in the group receiving low-dose statin (i.e., atorvastatin, $20 \mathrm{mg}$ ). These findings provide additional in vivo evidence that high-dose statins (i.e., $80 \mathrm{mg}$ ) might have a beneficial effect on plaque stability. Furthermore, these changes in USPIO-defined plaque inflammation could be observed within $6 \mathrm{wk}$, a relatively short treatment interval compared with the prolonged periods (years) that are required to observe changes in plaque burden. This study may also indicate that reductions in plaque inflammation may play an important role in the mechanism underlying the early beneficial effects of statins.

If adequately validated, USPIO-enhanced MRI methodology may be a useful imaging approach to access the therapeutic response to "antiinflammatory" interventions in patients with carotid atherosclerotic lesions. However, before USPIO-MRI may be routinely used for multicenter clinical testing, several issues with regard to the ATHEROMA study (114) need to be examined. The relatively small patient population limits the ability to generalize the dose response observed in that study. Although the authors found a weak correlation between the MRI data and the microemboli count on transcranial Doppler, the study still did not correlate the MRI findings to any hard clinical endpoints. In addition, aspects of USPIO-MRI quantification need to be addressed before this method can be used in large multicenter clinical trials. Differences in patient positioning, coil inhomogeneities, noise, and other artifacts may all induce signal loss that may not be indicative of USPIO uptake. Validation of semiquantitative analyses is needed, as well as improvements in imaging, including the use of positive contrast or white-marker data acquisition (gradient echo acquisition for superparamagnetic particles, inversion recovery on, ultrashort echo time, etc.), which may be acquired within the same imaging session, to improve image interpretation and data analysis (61).
The USPIO (ferumoxtran-10; Sinerem [Guerbet, LLC]) used in ATHEROMA is currently not approved by the Food and Drug Administration and is considered investigational. Sinerem was originally developed as a contrast agent for the lymphatics and bone marrow (115); as a result, high lymphatic uptake is expected. Because the signal loss observed by USPIOs is caused by dephasing of diffusing water protons, blooming effects (or signal loss over a larger distance) may be observed. Because of the proximity of the lymphatics to the arterial wall, the data obtained using the quadrant analysis approach may become biased or skewed by lymphatic tissue included in any given quadrant. Imaging of inflammatory changes using USPIO also requires 2 scans-a precontrast scan and a postcontrast infusion scan-at each imaging time point.

\section{Coronary CT Angiography (CTA) to Identify Unstable Plaque}

It was observed many years ago that coronary plaques that are prone to rupture are not associated with critical stenosis on contrast coronary angiography. Fluoroscopic coronary angiography is limited to showing the outline of the coronary lumen only. Intravascular ultrasound and coronary CTA image the cross section of the entire vessel, including the vessel wall and the lumen. These technologies provide in vivo information on plaque morphology. Several intravascular ultrasound studies have shown segmental dilation of the coronary vessel associated with large accumulation of low-acoustic-density material in the neointima consistent with large lipid cores. These large plaques do not encroach on the lumen because of the positive remodeling of the vessel wall (116-118). The process of segmental vessel remodeling, whether it is primary or secondary, is associated with plaque vulnerability.

Intravascular ultrasound studies are invasive procedures performed on patients with symptoms warranting catheterization and therefore not potentially useful for screening of high-risk patients. CTA is an imaging modality that can also provide cross-sectional views of the coronary vessel, assessing both the vessel wall and the lumen. In a recent study, investigators interpreted coronary CT angiogram findings for over 1,000 patients and followed the patients for coronary events. Positive vessel remodeling and lowattenuation plaques were used as the criteria for plaque vulnerability. The study found that patients with positively remodeled coronary segments with low-attenuation plaques were at higher risk of acute coronary syndrome than were patients without these findings (119). Coronary CTA involves the administration of iodinated contrast and radiation exposure (as does nuclear imaging) and slow heart rates. Because of the risks associated with radiation exposure, current American Heart Association/American College of Cardiology guidelines do not recommend CTA as a general screening tool in low-risk, asymptomatic patients. However, newer multislice CT scanners will make procedures shorter and simpler to perform and increase the 
potential of this technology as a screening procedure in high-risk groups.

\section{CHALLENGES FOR TRANSLATION}

For practical reasons, most studies on imaging vessel wall biology are initially performed on small animals. Advantages include the availability of established models of human vascular disease, experimental models with shorter time frames, and low cost. In addition to studying the pathophysiology of human disease, these small-animal imaging studies can help advance basic research and drug development and serve as a first step in the validation and screening of novel therapies.

Despite these advantages, there are also several challenges for successful translation of the preclinical findings to humans. The first challenge is that, despite the many similarities in vascular biology between humans and rodents, there are also differences, for example, MMP expression, which may ultimately affect translation to human disease. Human pathology is often more complex than simple models in small animals, complicating the interpretation of imaging studies.

A second challenge to clinical translation is the design of the imaging probes themselves. Achieving sufficient targetto-background levels for visualization on in vivo images requires a high number of binding sites or a radiochemical design to boost the signal at the target. Moreover, it is also important that the probe clear rapidly enough from the blood pool to reduce background levels yet remain in the circulation long enough to achieve binding. Uptake in nontarget organs must also be minimized. Another issue is that many of these probes are validated in mouse models using murine antibodies or antibody fragments. For human studies, especially when the probe might be injected at multiple time points to follow therapeutic efficacy, the potential exists for an immunogenic reaction that could adversely affect the binding of the probe to the intended target or, even worse, be unsafe.

The translation of complex nanoparticle technologies to clinical trials and ultimately to clinical practice also has its share of unique challenges. By far the greatest challenge is the failure of pharmaceutical and biomedical imaging companies to embrace nanotechnology, in part because each lacks the expertise of the other. From the drug development perspective, nanoparticles cannot go into the clinic until concerns about nanoparticle safety, including acute host immune or complement responses and the metabolism and elimination of the particle and its constituents, are satisfactorily addressed. These technical challenges of nanotechnology can be addressed by focused advancements in engineering and chemical designs. The developmental expertise to achieve these goals rests with the major pharmaceutical and bioimaging companies.

A third challenge for clinical translation is instrumentation. The numerous inherent difficulties of detecting and resolving minute regions of interest within a large field of view, and the presentation of these identified pixels in an easy-to-interpret and quantifiable manner, are unprecedented challenges. Hybrid imaging systems such as SPECT or PET/CT and PET/MRI are playing an important role in helping to localize hot-spot radiotracers within the vasculature. For practical purposes, much of the research work in this field has been performed in peripheral large arteries, such as the aorta and carotid arteries. Imaging smaller coronary vessels with radiotracer probes is complicated by the fact that the diameter of these vessels is below the spatial resolution of most SPECT and PET cameras. Although recent studies have demonstrated that vulnerable lesions can be imaged with high enough focal activity and low background myocardial activity, future advances in instrumentation resulting in higher spatial resolution and increased sensitivity will be helpful. Imaging the coronary arteries is further complicated by both cardiac and respiratory motion. Thus, it will be important to correct for these motion artifacts, especially when examining small coronary lesions.

Lastly, the cost of tracer development for vascular diseases may be prohibitive. This challenge may be at least partially overcome by developing tracers that are useful for multiple applications.

\section{CONCLUSION}

Advances in molecular biology, development of genetically altered mice, and careful observation of human pathologic specimens have produced a picture of the biologic and anatomic initiation and progression of atherosclerosis. This complex picture presents targets for the development of probes that, coupled with rapid advances in technology for both small-animal and clinical hybrid SPECT, PET, and MRI platforms, has broadened capabilities for both preclinical research and clinical imaging. Vascular remodeling manifests as either expansive or restrictive, and changes in the vessel wall composition (hypertrophy or hypotrophy) are common to all vascular pathologies. Enzymes involved in dissolving the extracellular matrix and proliferating cells comprising the neointima can be targeted for imaging. Inflammation is an important component of atherosclerosis. A positron-labeled probe, ${ }^{18} \mathrm{~F}$-FDG, is widely available for tumor imaging and shows promise as a marker of inflammatory activity of atherosclerotic plaque and plaque burden. It is being tested as a surrogate endpoint in drug trials. Experimental studies have shown that a single photon-labeled probe that binds the LOX-1 LDL receptor, a scavenger receptor on macrophages for oxidized LDL, is taken up in atherosclerotic lesions. This radiolabeled probe shows promise as an agent for imaging inflammation in atherosclerosis. Radiolabeled MPIs that target both inflammation and remodeling show promise in preclinical experiments. MRI alone offers information on anatomy and plaque composition and can 
be combined with imaging probes that target biologic processes. Nanoparticles with paramagnetic properties have been designed to target angiogenesis, which is an important process in advanced atherosclerotic plaque leading to intraplaque hemorrhage and instability. Iron-based particles, USPIOs, are taken up by macrophages in atheroma, and USPIO-MRI has the potential to become an approach to image inflamed and active atherosclerotic plaques with further refinements in acquisition parameters. Coronary CTA can detect 2 important features of coronary plaque vulnerability: large, soft plaque and focal vascular remodeling. All of these approaches show promise for imaging many of the known manifestations of atherosclerotic plaque instability, but application in the clinic will require the availability of nontoxic low-molecular-weight probes, imaging platforms, and demonstration of cost-effectiveness.

\section{REFERENCES}

1. Pasterkamp G, Fitzgerald PF, de Kleijn DP. Atherosclerotic expansive remodeled plaques: a wolf in sheep's clothing. J Vasc Res. 2002;39:514-523.

2. Johnson LL, Schofield LM, Verdesca SA, et al. In vivo uptake of radiolabeled antibody to proliferating smooth muscle cells in a swine model of coronary stent restenosis. J Nucl Med. 2000;41:1535-1540.

3. Sadeghi MM, Krassilnikova S, Zhang J, et al. Detection of injury-induced vascular remodeling by targeting activated alphavbeta 3 integrin in vivo. Circulation. 2004;110:84-90.

4. Zhang J, Krassilnikova S, Gharaei AA, et al. Alphavbeta3-targeted detection of arteriopathy in transplanted human coronary arteries: an autoradiographic study. FASEB J. 2005;19:1857-1859.

5. Schafers M, Riemann B, Kopka K, et al. Scintigraphic imaging of matrix metalloproteinase activity in the arterial wall in vivo. Circulation. 2004; 109:2554-2559.

6. Zhang J, Nie L, Razavian M, et al. Molecular imaging of activated matrix metalloproteinases in vascular remodeling. Circulation. 2008;118:1953-1960.

7. Nie L, Razavian M, Zhang J, et al. Imaging matrix metalloproteinase activation to predict aneurysm expansion in vivo [abstract]. J Nucl Med. 2009;50:658.

8. Rosen JM, Butler SP, Meinken GE, et al. Indium-111-labeled LDL: a potential agent for imaging atherosclerotic disease and lipoprotein biodistribution. J Nucl Med. 1990;31:343-350.

9. Vallabhajosula S, Paidi M, Badimon JJ, et al. Radiotracers for low density lipoprotein biodistribution studies in vivo: technetium-99m low density lipoprotein versus radioiodinated low density lipoprotein preparations. $\mathrm{J} \mathrm{Nucl}$ Med. 1988;29:1237-1245.

10. Lees AM, Lees RS, Schoen FJ, et al. Imaging human atherosclerosis with ${ }^{99 m}$ Tc-labeled low density lipoproteins. Arteriosclerosis. 1988;8:461-470.

11. Hartung D, Petrov A, Haider N, et al. Radiolabeled monocyte chemotactic protein 1 for the detection of inflammation in experimental atherosclerosis. J Nucl Med. 2007;48:1816-1821.

12. Kircher MF, Grimm J, Swirski FK, et al. Noninvasive in vivo imaging of monocyte trafficking to atherosclerotic lesions. Circulation. 2008;117:388-395.

13. Nahrendorf M, Zhang H, Hembrador S, et al. Nanoparticle PET-CT imaging of macrophages in inflammatory atherosclerosis. Circulation. 2008;117:379-387.

14. Jialal I, Devaraj S. The role of oxidized low density lipoprotein in atherogenesis. J Nutr. 1996;126(4, suppl)1053S-1057S.

15. Steinberg D, Witztum JL. Lipoproteins and atherogenesis: current concepts. JAMA. 1990;264:3047-3052.

16. Kugiyama K, Kerns SA, Morrisett JD, Roberts R, Henry PD. Impairment of endothelium-dependent arterial relaxation by lysolecithin in modified lowdensity lipoproteins. Nature. 1990;344:160-162.

17. Gerrity RG. The role of the monocyte in atherogenesis: I. Transition of bloodborne monocytes into foam cells in fatty lesions. Am J Pathol. 1981;103:181-190.

18. Drake TA, Hannani K, Fei HH, Lavi S, Berliner JA. Minimally oxidized lowdensity lipoprotein induces tissue factor expression in cultured human endothelial cells. Am J Pathol. 1991;138:601-607.

19. Rahman MM, Varghese Z, Fuller BJ, Moorhead JF. Renal vasoconstriction induced by oxidized LDL is inhibited by scavengers of reactive oxygen species and L-arginine. Clin Nephrol. 1999;51:98-107.
20. Sawamura T, Kume N, Aoyama T, et al. An endothelial receptor for oxidized low-density lipoprotein. Nature. 1997;386:73-77.

21. Kume N, Murase T, Moriwaki H, et al. Inducible expression of lectin-like oxidized LDL receptor-1 in vascular endothelial cells. Circ Res. 1998;83:322327.

22. Li DY, Zhang YC, Philips MI, Sawamura T, Mehta JL. Upregulation of endothelial receptor for oxidized low-density lipoprotein (LOX-1) in cultured human coronary artery endothelial cells by angiotensin II type 1 receptor activation. Circ Res. 1999;84:1043-1049.

23. Murase T, Kume N, Korenaga R, et al. Fluid shear stress transcriptionally induces lectin-like oxidized LDL receptor-1 in vascular endothelial cells. Circ Res. 1998;83:328-333.

24. Yoshida H, Kondratenko N, Green S, Steinberg D, Quehenberger O. Identification of the lectin-like receptor for oxidized low-density lipoprotein in human macrophages and its potential role as a scavenger receptor. Biochem J. 1998;334:9-13.

25. Ishino S, Mukai T, Kuge Y, et al. Targeting of lectinlike oxidized low-density lipoprotein receptor 1 (LOX-1) with ${ }^{99 \mathrm{~m}} \mathrm{Tc}$-labeled anti-LOX-1 antibody: potential agent for imaging of vulnerable plaque. J Nucl Med. 2008;49:1677-1685.

26. Li D, Patel A, Klibanov A, Kramer, et al. Molecular imaging of atherosclerotic plaques targeted on oxidized LDL receptor LOX-1 using SPECT/CT and magnetic resonance [abstract]. J Nucl Med. 2009;50:657.

27. Virmani R, Kolodgie FD, Burke AP, Farb A, Schwartz SM. Lesions from sudden coronary death: a comprehensive morphology classification scheme for atherosclerotic lesions. Arterioscler Thromb Vasc Biol. 2000;20:1262-1275.

28. Varnava AM, Mills PG, Davies MJ. Relationship between coronary artery remodeling and plaque vulnerability. Circulation. 2002;105:939-943.

29. Kolodgie FD, Narula J, Burke AP, et al. Localization of apoptotic macrophages at the site of plaque rupture in sudden coronary death. Am J Pathol. 2000; 157:1259-1268.

30. Wolters SL, Corsten MF, Reutelingsperger CP, Narula J, Hofstra L. Cardiovascular molecular imaging of apoptosis. Eur J Nucl Med Mol Imaging. 2007;34(suppl 1):S86-S98.

31. Nguyen QD, Smith G, Glaser M, et al. Positron emission tomography imaging of drug-induced tumor apoptosis with a caspase-3/7 specific $\left[{ }^{18} \mathrm{~F}\right]$-labeled isatin sulfonamide. Proc Natl Acad Sci USA. 2009;106:16375-16380.

32. Blankenberg FG, Katsikis PD, Tait JF, et al. In vivo detection and imaging of phosphatidylserine expression during programmed cell death. Proc Natl Acad Sci USA 1998;95:6349-6354.

33. Hofstra L, Liem IH, Dumont EA, et al. Visualisation of cell death in vivo in patients with acute myocardial infarction. Lancet. 2000;356:209-212.

34. Kolodgie FD, Petrov A, Virmani R, et al. Targeting of apoptotic macrophages and experimental atheroma with radiolabeled annexin V: a technique with potential for noninvasive imaging of vulnerable plaque. Circulation. 2003; 108:3134-3139.

35. Isobe S, Tsimikas S, Zhou J, et al. Noninvasive imaging of atherosclerotic lesions in apolipoprotein E-deficient and low-density-lipoprotein receptordeficient mice with annexin A5. J Nucl Med. 2006;47:1497-1505.

36. Sarai M, Hartung D, Petrov A, et al. Broad and specific caspase inhibitorinduced acute repression of apoptosis in atherosclerotic lesions evaluated by radiolabeled annexin A5 imaging. J Am Coll Cardiol. 2007;50:2305-2312.

37. Johnson LL, Schofield L, Donahay T, Narula N, Narula J. ${ }^{99 \mathrm{~m}} \mathrm{Tc}$-annexin V imaging for in vivo detection of atherosclerotic lesions in porcine coronary arteries. J Nucl Med. 2005;46:1186-1193.

38. Newby AC. Dual role of matrix metalloproteinases (Matrixins) in intimal thickening and atherosclerotic plaque rupture. Physiol Rev. 2005;85:1-31.

39. Galis ZS, Sukhova GK, Lark MW, Libby P. Increased expression of matrix metalloproteinases and matrix degrading activity in vulnerable regions of human atherosclerotic plaques. J Clin Invest. 1994;94:2493-2503.

40. Sukhova GK, Schonbeck U, Rabkin E, et al. Evidence for increased collagenolysis by interstitial collagenases- 1 and -3 in vulnerable human atheromatous plaques. Circulation. 1999;99:2503-2509.

41. Sluijter JP, Pulskens WP, Schoneveld AH, et al. Matrix metalloproteinase 2 is associated with stable and matrix metalloproteinases 8 and 9 with vulnerable carotid atherosclerotic lesions a study in human endarterectomy specimen pointing to a role for different extracellular matrix metalloproteinase inducer glycosylation forms. Stroke. 2006;37:235-239.

42. Xue CB, Voss ME, Nelson D, et al. Design, synthesis, and structure-activity relationships of macrocyclic hydroxamic acids that inhibit tumor necrosis factor alpha release in vitro and in vivo. J Med Chem. 2001;44:2636-2640.

43. Ohshima S, Petrov A, Fujimoto S, et al. Molecular imaging of matrix metalloproteinase expression in atherosclerotic plaques of mice deficient in apolipoprotein E or low-density-lipoprotein receptor. J Nucl Med. 2009; 50:612-617. 
44. Fujimoto S, Hartung D, Edward DS, et al. Molecular imaging of matrix metalloproteinase in atherosclerotic lesions: resolution with dietary modification and statin therapy. J Am Coll Cardiol. 2008;52:1847-1857.

45. Deguchi JO, Aikawa $\mathrm{M}$, Tung $\mathrm{CH}$, et al. Inflammation in atherosclerosis: visualizing matrix metalloproteinase action in macrophages in vivo. Circulation. 2006;114:55-62.

46. Lancelot E, Amirbekian V, Brigger I, et al. Evaluation of matrix metalloproteinases in atherosclerosis using a novel noninvasive imaging approach. Arterioscler Thromb Vasc Biol. 2008;28:425-432.

47. Tekabe Y, Qing L, Luma J, et al. Noninvasive monitoring the biology of atherosclerotic plaque development with radiolabeled annexin $\mathrm{V}$ and matrix metalloproteinase inhibitor in spontaneous atherosclerotic mice [abstract]. Circulation. 2009;120(suppl):S365-S366.

48. Stark DD, Weissleder R, Elizondo G, et al. Superparamagnetic iron oxide: clinical application as a contrast agent for MR imaging of the liver. Radiology. 1988;168:297-301.

49. Weissleder R, Hahn PF, Stark DD, et al. Superparamagnetic iron oxide: enhanced detection of focal splenic tumors with MR imaging. Radiology. 1988;169:399-403.

50. Frank H, Weissleder R, Brady TJ. Enhancement of MR angiography with iron oxide: preliminary studies in whole-blood phantom and in animals. AJR. 1994;162:209-213.

51. Kresse M, Wagner S, Pfefferer D, et al. Targeting of ultrasmall superparamagnetic iron oxide (USPIO) particles to tumor cells in vivo by using transferrin receptor pathways. Magn Reson Med. 1998;40:236-242.

52. Jung CW, Jacobs P. Physical and chemical properties of superparamagnetic iron oxide MR contrast agents: ferumoxides, ferumoxtran, ferumoxsil. Magn Reson Imaging. 1995; 13:661-674.

53. Schmitz SA, Coupland SE, Gust R, et al. Superparamagnetic iron oxideenhanced MRI of atherosclerotic plaques in Watanabe hereditable hyperlipidemic rabbits. Invest Radiol. 2000;35:460-471.

54. Schmitz SA, Taupitz M, Wagner S, et al. Magnetic resonance imaging of atherosclerotic plaques using superparamagnetic iron oxide particles. J Magn Reson Imaging. 2001;14:355-361.

55. Schmitz SA, Taupitz M, Wagner S, et al. Iron-oxide-enhanced magnetic resonance imaging of atherosclerotic plaques: postmortem analysis of accuracy, inter-observer agreement, and pitfalls. Invest Radiol. 2002;37:405-411.

56. Ruehm SG, Corot C, Vogt P, Kolb S, Debatin JF. Magnetic resonance imaging of atherosclerotic plaque with ultrasmall superparamagnetic particles of iron oxide in hyperlipidemic rabbits. Circulation. 2001;103:415-422.

57. Kooi ME, Cappendijk VC, Cleutjens KB, et al. Accumulation of ultrasmall superparamagnetic particles of iron oxide in human atherosclerotic plaques can be detected by in vivo magnetic resonance imaging. Circulation. 2003;107:2453-2458.

58. Herborn CU, Vogt FM, Lauenstein TC, et al. Magnetic resonance imaging of experimental atherosclerotic plaque: comparison of two ultrasmall superparamagnetic particles of iron oxide. J Magn Reson Imaging. 2006;24:388-393.

59. Cunningham $\mathrm{CH}$, Arai $\mathrm{T}$, Yang $\mathrm{P}$, et al. Positive contrast magnetic resonance imaging of cells labeled with magnetic nanoparticles. Magn Reson Med. 2005;53:999-1005.

60. Dharmakumar R, Koktzoglou I, Li D. Generating positive contrast from offresonant spins with steady-state free precession magnetic resonance imaging: theory and proof-of-principle experiments. Phys Med Biol. 2006;51:4201-4215.

61. Mani V, Briley-Saebo KC, Itskovich VV, Samber DD, Fayad ZA. Gradient echo acquisition for superparamagnetic particles with positive contrast (GRASP): sequence characterization in membrane and glass superparamagnetic iron oxide phantoms at 1.5T and 3T. Magn Reson Med. 2006;55:126-135.

62. Zurkiya $\mathrm{O}, \mathrm{Hu} \mathrm{X}$. Off-resonance saturation as a means of generating contrast with superparamagnetic nanoparticles. Magn Reson Med. 2006;56:726-732.

63. Stuber M, Gilson WD, Schar M, et al. Positive contrast visualization of iron oxide-labeled stem cells using inversion-recovery with ON-resonant water suppression (IRON). Magn Reson Med. 2007;58:1072-1077.

64. Korosoglou G, Tang L, Kedziorek D, et al. Positive contrast MR-lymphography using inversion recovery with ON-resonant water suppression (IRON). J Magn Reson Imaging. 2008;27:1175-1180.

65. Korosoglou G, Weiss RG, Kedziorek DA, et al. Noninvasive detection of macrophage-rich atherosclerotic plaque in hyperlipidemic rabbits using "positive contrast" magnetic resonance imaging. J Am Coll Cardiol. 2008;52:483-491.

66. Korosoglou G, Shah S, Vonken EJ, et al. Off-resonance angiography: a new method to depict vessels — phantom and rabbit studies. Radiology. 2008;249:501-509.

67. Dunn JF, Roche MA, Springett R, et al. Monitoring angiogenesis in brain using steady-state quantification of DeltaR2 with MION infusion. Magn Reson Med. 2004;51:55-61.

68. Hogemann D, Josephson L, Weissleder R, Basilion JP. Improvement of MRI probes to allow efficient detection of gene expression. Bioconjug Chem. 2000;11:941-946.
69. Caruthers S, Senpan A, Pan D, et al. A novel targeted iron oxide nanocolloid agent for rapid detection of fibrin clots via T1 and T2 weighted MRI [abstract]. J Cardiovasc Magn Reson. 2008;10(suppl 1):A384.

70. Sipkins DA, Cheresh DA, Kazemi MR, et al. Detection of tumor angiogenesis in vivo by alphaVbeta3-targeted magnetic resonance imaging. Nat Med. 1998;4:623-626.

71. Lanza GM, Lorenz C, Fischer S, et al. Enhanced detection of thrombi with a novel fibrin-targeted magnetic resonance imaging agent. Acad Radiol. 1998; 5(suppl 1):s173-s176.

72. Botnar RM, Buecker A, Wiethoff AJ, et al. In vivo magnetic resonance imaging of coronary thrombosis using a fibrin-binding molecular magnetic resonance contrast agent. Circulation. 2004;110:1463-1466.

73. Mulder WJ, Strijkers GJ, Habets JW, et al. MR molecular imaging and fluorescence microscopy for identification of activated tumor endothelium using a bimodal lipidic nanoparticle. FASEB J. 2005;19:2008-2010.

74. Mulder WJ, Strijkers GJ, van Tilborg GA, Griffioen AW, Nicolay K. Lipidbased nanoparticles for contrast-enhanced MRI and molecular imaging. NMR Biomed. 2006;19:142-164.

75. Mulder WJ, van der Schaft DW, Hautvast PA, et al. Early in vivo assessment of angiostatic therapy efficacy by molecular MRI. FASEB J. 2007;21:378-383.

76. Frias JC, Williams KJ, Fisher EA, Fayad ZA. Recombinant HDL-like nanoparticles: a specific contrast agent for MRI of atherosclerotic plaques. J Am Chem Soc. 2004;126:16316-16317.

77. Lipinski MJ, Amirbekian V, Frias JC, et al. MRI to detect atherosclerosis with gadolinium-containing immunomicelles targeting the macrophage scavenger receptor. Magn Reson Med. 2006;56:601-610.

78. Yu X, Song S-K, Chen J, et al. High-resolution MRI characterization of human thrombus using a novel fibrin-targeted paramagnetic nanoparticle contrast agent. Magn Reson Med. 2000;44:867-872.

79. Flacke S, Fischer S, Scott M, et al. A novel MRI contrast agent for molecular imaging of fibrin: implications for detecting vulnerable plaques. Circulation. 2001; 104:1280-1285.

80. Winter P, Chen J, Song S-K, et al. Relaxivities of paramagnetic nanoparticle contrast agents for targeted molecular imaging [abstract]. Proc Intl Soc Mag Reson Med. 2001;9:54.

81. Winter PM, Caruthers S, Yu X, et al. Improved molecular imaging contrast agent for detection of human thrombus. Magn Reson Med. 2003;50:411416 .

82. Winter PM, Neubauer A, Caruthers S, et al. Endothelial alpha(nu)beta(3)integrin targeted fumagillin nanoparticles inhibit angiogenesis in atherosclerosis. Arterioscler Thromb Vasc Biol. 2006;26:2103-2109.

83. Winter P, Caruthers S, Zhang H, et al. Antiangiogenic synergism of integrintargeted fumagillin nanoparticles and atorvastatin in atherosclerosis. $J$ Am Coll Cardiol Imaging. 2008;1:624-634.

84. Moreno PR, Purushothaman KR, Fuster V, et al. Plaque neovascularization is increased in ruptured atherosclerotic lesions of human aorta: implications for plaque vulnerability. Circulation. 2004;110:2032-2038.

85. Moreno PR, Purushothaman KR, Sirol M, Levy AP, Fuster V. Neovascularization in human atherosclerosis. Circulation. 2006;113:2245-2252.

86. Koutouzis M, Nomikos A, Nikolidakis S, et al. Statin treated patients have reduced intraplaque angiogenesis in carotid endarterectomy specimens. Atherosclerosis. 2007;192:457-463.

87. Kolodgie F, Narula J, Yuan C, Finn A, Gold H, Virmani R. Eliminating plaque angiogenesis [reply]. J Am Coll Cardiol. 2007;50:1521.

88. Virmani R, Kolodgie FD, Burke AP, et al. Atherosclerotic plaque progression and vulnerability to rupture: angiogenesis as a source of intraplaque hemorrhage. Arterioscler Thromb Vasc Biol. 2005;25:2054-2061.

89. Winter PM, Morawski AM, Caruthers SD, et al. Molecular imaging of angiogenesis in early-stage atherosclerosis with alpha(v)beta3-integrin-targeted nanoparticles. Circulation. 2003;108:2270-2274.

90. Cyrus T, Abendschein DR, Caruthers SD, et al. MR three-dimensional molecular imaging of intramural biomarkers with targeted nanoparticles. J Cardiovasc Magn Reson. 2006;8:535-541.

91. Cyrus T, Zhang H, Allen JS, et al. Intramural delivery of rapamycin with alphavbeta3-targeted paramagnetic nanoparticles inhibits stenosis after balloon injury. Arterioscler Thromb Vasc Biol. 2008;28:820-826.

92. Feuerlein S, Roessl E, Proksa R, et al. Multienergy photon-counting K-edge imaging: potential for improved luminal depiction in vascular imaging. Radiology. 2008;249:1010-1016.

93. Schlomka JP, Roessl E, Dorscheid R, et al. Experimental feasibility of multienergy photon-counting K-edge imaging in pre-clinical computed tomography. Phys Med Biol. 2008;53:4031-4047.

94. Roessl E, Proksa R. K-edge imaging in x-ray computed tomography using multi-bin photon counting detectors. Phys Med Biol. 2007;52:4679-4696. 
95. Roessl E, Ziegler A, Proksa R. On the influence of noise correlations in measurement data on basis image noise in dual-energylike x-ray imaging. Med Phys. 2007;34:959-966.

96. Calcagno C, Cornily JC, Hyafil F, et al. Detection of neovessels in atherosclerotic plaques of rabbits using dynamic contrast enhanced MRI and ${ }^{18}$ F-FDG PET. Arterioscler Thromb Vasc Biol. 2008;28:1311-1317.

97. Kerwin WS, Oikawa M, Yuan C, Jarvik GP, Hatsukami TS. MR imaging of adventitial vasa vasorum in carotid atherosclerosis. Magn Reson Med. 2008;59: 507-514.

98. Fayad ZA, Razzouk L, Briley-Saebo KC, Mani V. Iron oxide magnetic resonance imaging for atherosclerosis therapeutic evaluation: still "rusty?" $J$ Am Coll Cardiol. 2009;53:2051-2052.

99. Tang TY, Howarth SP, Miller SR, Graves MJ, et al. The ATHEROMA (Atorvastatin Therapy: Effects on Reduction of Macrophage Activity) study: evaluation using ultrasmall superparamagnetic iron oxide-enhanced magnetic resonance imaging in carotid disease. J Am Coll Cardiol. 2009;53:2039-2050.

100. Ridker PM, Danielson E, Fonseca FA, et al. Reduction in C-reactive protein and LDL cholesterol and cardiovascular event rates after initiation of rosuvastatin: a prospective study of the JUPITER trial. Lancet. 2009;373:1175-1182.

101. Sanz J, Fayad ZA. Imaging of atherosclerotic cardiovascular disease. Nature. 2008;451:953-957.

102. Yun M, Cotter A, Kim W, et al. F-18 FDG uptake in the large arteries: correlation with the risk factors of atherosclerosis [abstract]. J Nucl Med. 2001;42(suppl):2P.

103. Yun M, Jang S, Cucchiara A, Newberg AB, Alavi A. F-18 FDG uptake in the large arteries: a correlation study with the atherogenic risk factors. Semin Nucl Med. 2002;32:70-76.

104. Bural GG, Torigian DA, Chamroonrat W, et al. FDG-PET is an effective imaging modality to detect and quantify age-related atherosclerosis in large arteries. Eur J Nucl Med Mol Imaging. 2008;35:562-569.

105. Ben-Haim S, Kupzov E, Tamir A, Israel O. Evaluation of ${ }^{18}$ F-FDG uptake and arterial wall calcifications using ${ }^{18}$ F-FDG PET/CT. J Nucl Med. 2004;45: 1816-1821.

106. Dunphy MP, Freiman A, Larson SM, Strauss HW. Association of vascular ${ }^{18} \mathrm{~F}-$ FDG uptake with vascular calcification. J Nucl Med. 2005;46:1278-1284.

107. Tatsumi M, Cohade C, Nakamoto Y, Wahl RL. Fluorodeoxyglucose uptake in the aortic wall at PET/CT: possible finding for active atherosclerosis. Radiology. 2003;229:831-837.
108. Rudd JH, Warburton EA, Fryer TD, Jones HA, et al. Imaging atherosclerotic plaque inflammation with $\left[{ }^{18} \mathrm{~F}\right]$-fluorodeoxyglucose positron emission tomography. Circulation. 2002;105:2708-2711.

109. Wu YW, Kao HL, Chen MF, et al. Characterization of plaques using ${ }^{18}$ F-FDG $\mathrm{PET} / \mathrm{CT}$ in patients with carotid atherosclerosis and correlation with matrix metalloproteinase-1. J Nucl Med. 2007;48:227-233.

110. Tahara N, Kai H, Yamagishi S, et al. Vascular inflammation evaluated by $\left[{ }^{18} \mathrm{~F}\right]-$ fluorodeoxyglucose positron emission tomography is associated with the metabolic syndrome. J Am Coll Cardiol. 2007;49:1533-1539.

111. Tahara N, Kai H, Ishibashi M, et al. Simvastatin attenuates plaque inflammation: evaluation by fluorodeoxyglucose positron emission tomography. J Am Coll Cardiol. 2006;48:1825-1831.

112. Lee SJ, On YK, Lee EJ, et al. Reversal of vascular ${ }^{18}$ F-FDG uptake with plasma high-density lipoprotein elevation by atherogenic risk reduction. $\mathrm{J}$ Nucl Med. 2008;49:1277-1282.

113. Wykrzykowska J, Lehman S, Williams G, et al. Imaging of inflamed and vulnerable plaque in coronary arteries with ${ }^{18} \mathrm{~F}-\mathrm{FDG}$ PET/CT in patients with suppression of myocardial uptake using a low-carbohydrate, high-fat preparation. J Nucl Med. 2009;50:563-568.

114. Schwartz GG, Olsson AG, Ezekowitz MD, et al. Effects of atorvastatin on early recurrent ischemic events in acute coronary syndromes: The MIRACL study-a randomized controlled trial. JAMA. 2001;285:1711-1718.

115. Bellin MF, Roy C, Kinkel K, et al. Lymph node metastases: safety and effectiveness of MR imaging with ultrasmall superparamagnetic iron oxide particles-initial clinical experience. Radiology. 1998;207:799-808.

116. Schoenhagen P, Ziada KM, Kapadia SR, et al. Extent and direction of arterial remodeling in stable versus unstable coronary syndromes. Circulation. 2000;101: 598-603.

117. Nakamura M, Nishikawa H, Mukai S, et al. Impact of coronary artery remodeling on clinical presentation of coronary artery disease: an intravascular ultrasound study. J Am Coll Cardiol. 2001;37:63-69.

118. Takeuchi H, Morino Y, Matsukage T, et al. Impact of vascular remodeling on the coronary plaque compositions: an investigation with in vivo tissue characterization using integrated backscatter-intravascular ultrasound. Atherosclerosis. 2009;202:476-482.

119. Motoyama S, Sarai M, Harigaya H, et al. Computed tomographic angiography characteristics of atherosclerotic plaques subsequently resulting in acute coronary syndrome. J Am Coll Cardiol. 2009;54:49-57. 